\title{
Linear Extraction of Satellite Imageries using Mathematical Morphology
}

\author{
Ms.Neeti Daryal \\ Department of Computer Sc \& Appls \\ M L N College, Yamuna Nagar
}

\author{
Dr. Vinod Kumar \\ Department of Mathematics \\ J.V. Jain College,Sharanpur
}

\begin{abstract}
The methodology used in this paper is based on the use of morphological operators contained in toolbox of Mathematical Morphology developed in the software MATLAB. The application of routines in image processing is aimed initially to improve the visual quality of the features of interest in digital grayscale images, which will then afterwards be extracted. Increasingly seeking to get improvement in quality of the extracted feature, the image was binarized through the binary operator with threshold. Methodology adapted to skeleton scanned images using Mathematical Morphology from remotely sensed data is a challenging issue in the field of digital image processing. Image skeletonization is one of the many morphological image processing operations. skeletonization is very often an intermediate step towards object recognition, it should have a low computational cost. Literature about above said methodology is also mentioned in this paper.
\end{abstract}

\section{General Terms}

Dilation, erosion, gray scale, image analysis, mathematical morphology, opening, and thresholding.

\section{Keywords}

Keywords are your own designated keywords which can be used for easy location of the manuscript using any search engines.

\section{INTRODUCTION}

Mathematical Morphology is a method for quantitative analysis of spatial structures that aims at analyzing shapes and forms of an object.

Extensive research has been done on satellite imagery. In this paper we have chosen to show the application of the skeltonization skeletinization in the field of remote sensing and are described how linear features can be extracted from satellite imageries. The methodology for linear feature extraction from satellite imageries may be consists of three steps: Preprocessing, Thinning and Postprocessing.

The procedure of Pre-processing is to facilitate the extract of the feature of satellite imageries. Preprocessing involves two steps: grayscale opening and thresholding. Erosion, in which pixels matching a given pattern are deleted from the image and Dilation, in which a small area around the pixel is set to a given pattern. Thresholding can be implemented though Otsu's method which is used to automatically perform histogram shapebased image or, the reduction of a graylevel image to a binary image. The algorithm assumes that the image to be thresholded contains two classes of pixels foreground and background then calculates the optimum threshold separating those two classes so that their combined spread (intra-class variance) is minimal. In next step Skeletonization is performed through thinning because Skeletonization is especially suited to the processing of binary images or grayscale images.

The Skeletonization results are imported to MATLAB to obtain desire results. Results are shown Literature about the above mentioned methods is also included in this paper.

\section{Objectives}

The general aims of this research are to present the results obtained from the use of Mathematical Morphology in the extraction and treatment of lineal features in images, seeking to aid the processes of cartographic products updating. Also, to verify the potentiality of the tools of mathematical morphology available in the toolbox of Mathematical Morphology couple to the software MATLAB, through the application of routines associated with the task of extracting of features of interest.

\section{Methodology}

For the extraction of the features of interest were applied routines of mathematical morphology on satellite images. The software MATLAB 7.0 was used as platform for the toolbox of Mathematical Morphology, which contains the used operators. The operators were applied on the image with the aim of testing their efficiency later in the obtaining results obtained, which can be used, in the updating of cartographic products.

The methodology is split into the following three steps:

\section{Preprocessing \\ 2. Thinning \\ 3. Post processing.}

\subsection{Preprocessing}

In the pre-processing stage, the operators used are: grayscale opening and thresholding [4]. This stage consisted of several tests where, in each one, new parameters were used so that in the end of the process the best possible results were obtained.

First grayscale opening is performed on the image. Grayscale opening is done in two steps:

- grayscale erosion

- $\quad$ grayscale dilation on the same structuring element [15]. Secondly Then thresholding is done using Otsu's algorithm which results in a binary image.

\subsubsection{Dilation}

Dilation is one of the basic operators in the area of mathematical morphology. The basic effect of the operator on a binary image is 
to gradually enlarge the boundaries of regions of foreground pixels [15] (see figure 1) shows the example in which a shape (in blue) and its morphological dilation (in green) and erosion (in yellow) [1].

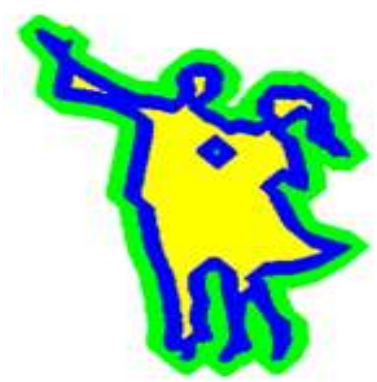

"Figure 1" Example of Shape, Erosion and dilation

Thus areas of foreground pixels grow in size while holes within those regions become smaller (see figure 2[3]).
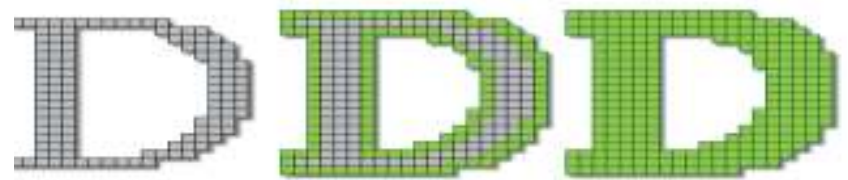

“Figure 2" Dilation

\subsubsection{Erosion}

Erosion is one of the basic operators in the area of mathematical morphology. The basic effect of the operator on a binary image is to erode away the boundaries of regions of foreground pixels. Thus areas of foreground pixels shrink in size, and holes within those areas become larger [15] (see figure 3[3]).
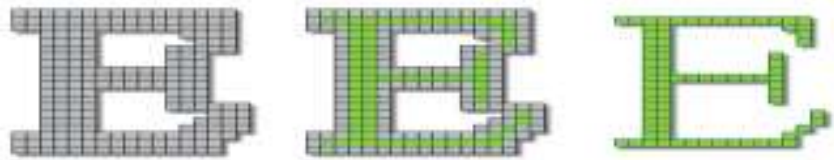

"Figure 3" Erosion

An example of grayscale opening (see figure 5) where the scanned image (see figure 4[4]).

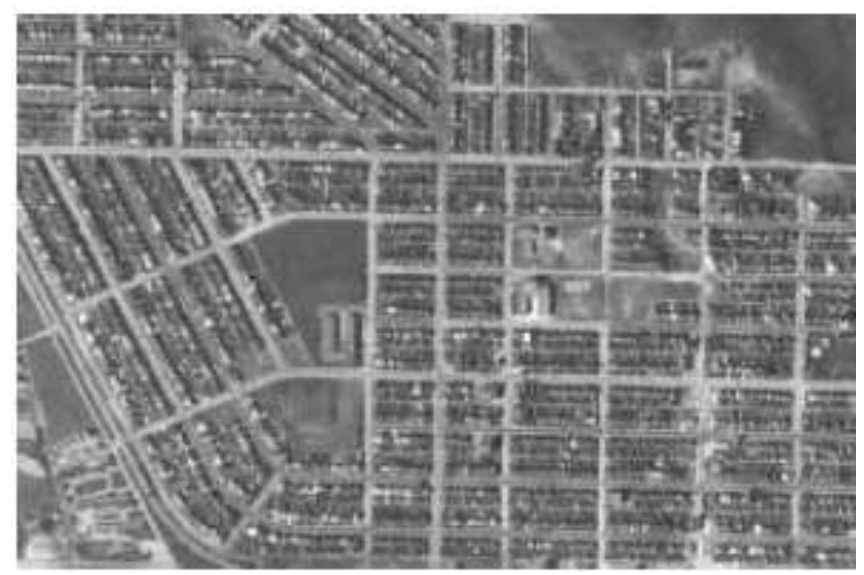

"Figure 4" Scanned Satellite Image

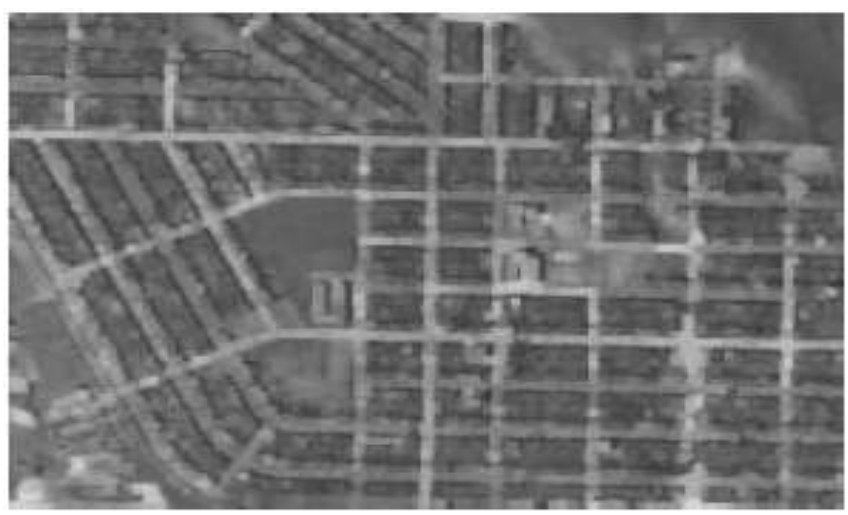

"Figure 5" Grayscale Opening

\subsubsection{Thresholding}

Thresholding is used to separate the regions of the image corresponding to an object from its background [7]. Thresholding is a very convenient way of performing segmentation. Here, we use the difference in intensities or colors in the foreground and background regions of an image to generate a binary image [16] and [15]. Thus thresholding can be defined as an image processing function that is used to segment an image by setting all pixels whose intensity values are above a threshold to a foreground value and the remaining pixels to a background value [16]. It can also be used to see what areas of an image consist of pixels whose values lie within a specified range, or band of intensities (or colors). The input to a thresholding operation is typically a grayscale or color image [8].

In Otsu's method we exhaustively search for the threshold that minimizes the intra-class variance, defined as a weighted sum of variances of the two classes:

$\sigma_{w}^{2}(t)=\omega_{1}(t) \sigma_{1}^{2}(t)+\omega_{2}(t) \sigma_{2}^{2}(t)$

Weights $\omega_{i}$ are the probabilities of the two classes separated by a threshold $t$ and $\sigma_{i}^{2}$ variances of these classes.

Otsu shows that minimizing the intra-class variance is the same as maximizing inter-class variance [4]:

$\sigma_{b}^{2}(t)=\sigma^{2}-\sigma_{w}^{2}(t)=\omega_{1}(t) \omega_{2}(t)\left[\mu_{1}(t)-\mu_{2}(t)\right]^{2}$

which is expressed in terms of class probabilities $\omega_{i}$ and class means $\mu_{i}$ which in turn can be updated iteratively. This idea yields an effective algorithm (see figure 6, figure 7).

\subsubsection{Algorithm}

1. Compute histogram and probabilities of each intensity level

2. Set up initial $\omega_{i}(0)$ and $\mu_{i}(0)$

3. Step through all possible thresholds $t=1 \ldots$ maximum intensity

1. Update $\omega_{i}$ and $\mu_{i}$ 


\section{Compute $\sigma_{b}^{2}(t)$}

4. Desired threshold corresponds to the maximum $\sigma_{b}^{2}(t)$

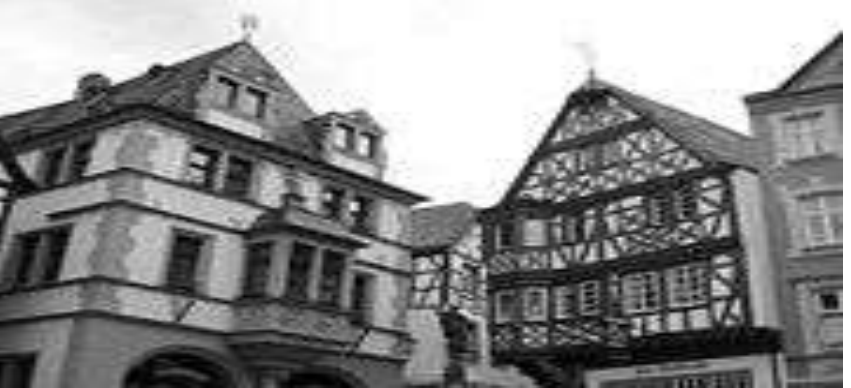

"Figure 6" Original Image

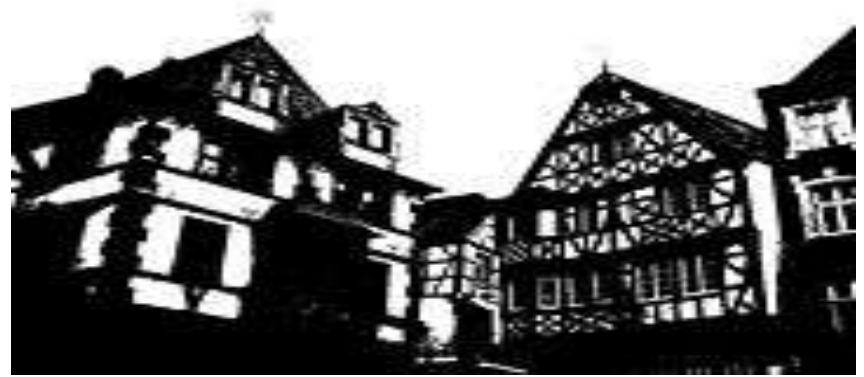

"Figure 7" Thresholding using Otsu's algorithm

\subsection{Thinning}

In this paper Thinning is done using Skeletonization by Mathematical Morphology[7]. Thinning is one of the Mathematical Morphological operations that are particularly used for skeletonization [15]. There are a variety of parallel thinning algorithms available in the field of digital image processing.

We have chosen to use Zhang-Suen's algorithm for thinning because it has been used as a basis for comparison of thinning methods for many years and it is comparatively fast and simple to implement [15].

Zhang-Suen's algorithm for thinning is a parallel method, meaning that the new value for any pixel can be computed only by using the values obtained from the previous iteration. The algorithm is divided into two sub iterations. In one sub iteration, a pixel $I(i, j)$ is deleted (or marked for deletion) (see figure 8 and figure 9) if the following four conditions are all true:

1. Its connectivity number is one.

2. It has at least two black neighbors and not more than six.

3. At least one of $I(i, j+1), I(i-1, j)$ and $I(i, j-1)$ are background (white).

4. At least one of $I(i-1, j), I(i+1, j)$ and $I(i, j-1)$ are background.
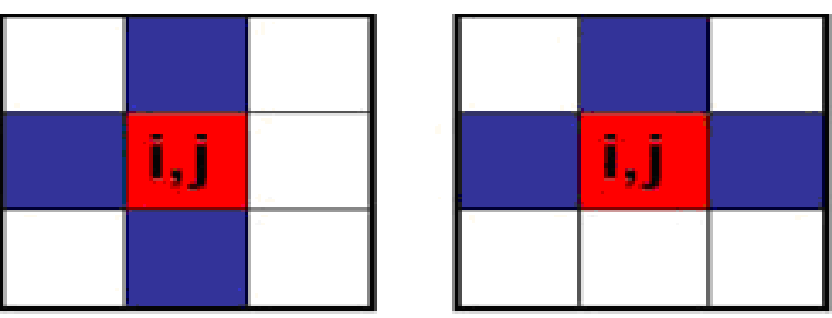

"Figure 8" Pixel marked for deletion in $\mathbf{1}^{\text {st }}$ iteration

At the end of this sub iteration the marked pixels are deleted. In the next sub iteration, a pixel $I(i, j)$ is deleted (or marked for deletion) if the following four conditions are all true:

1. Its connectivity number is one.

2. It has at least two black neighbors and not more than six.

3. At least one of $I(i-1, j), I(i, j+1)$ and $I(i+1, j)$ are background.

4. At least one of $I(i, j+1), I(i+1, j)$ and $I(i, j-1)$ are background.
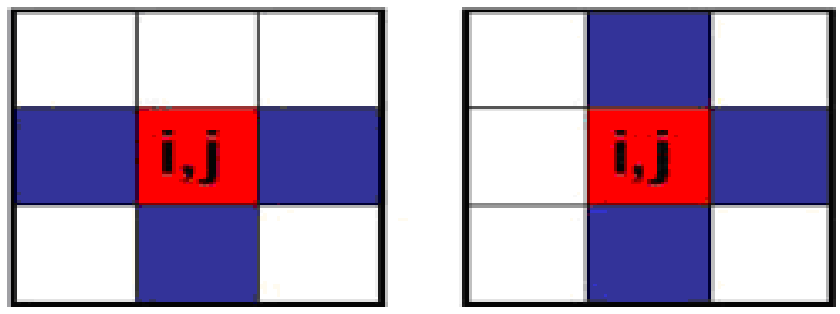

"Figure 9" Pixel marked for deletion in $2^{\text {nd }}$ iteration

The marked pixels are deleted. If at the end of either sub iteration there are no pixels to be deleted, then the skeleton is complete and the program stops [15].

The result of Skeletonization by applying above algorithm of Mathematical Morphology on figure 5 in MATLAB is shown in figure $10[6]$ and figure 11 respectively.

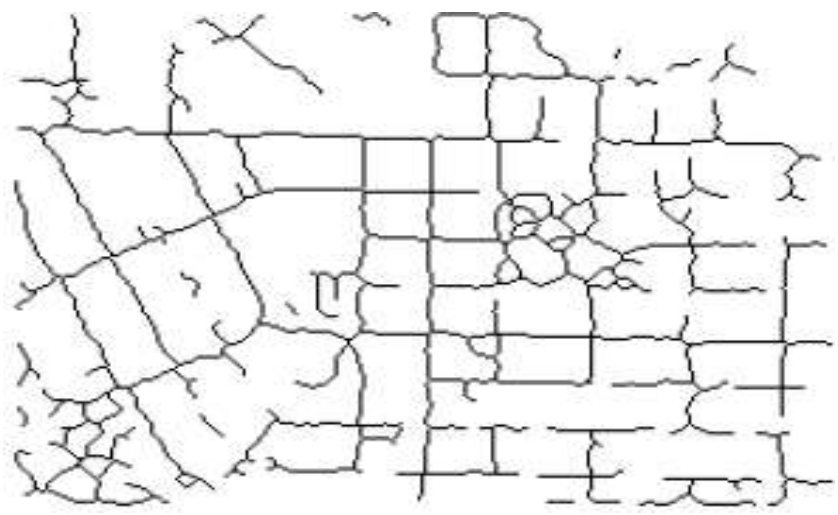

"Figure 10" Skeletonization by Mathematical Morphology

\subsection{Postprocessing}

The skeletonized raster image obtained by using Mathematical Morphology is imported into MATLAB. Then it is converted to vector data. 


\section{Result}

The results of extracting linear features on two other grayscale satellite images are shown below. Figure 11[10] is the Scanned image and its corresponding results are shown in figure 12 . Figure 13 is the second image and its Corresponding results are shown in figure 14.

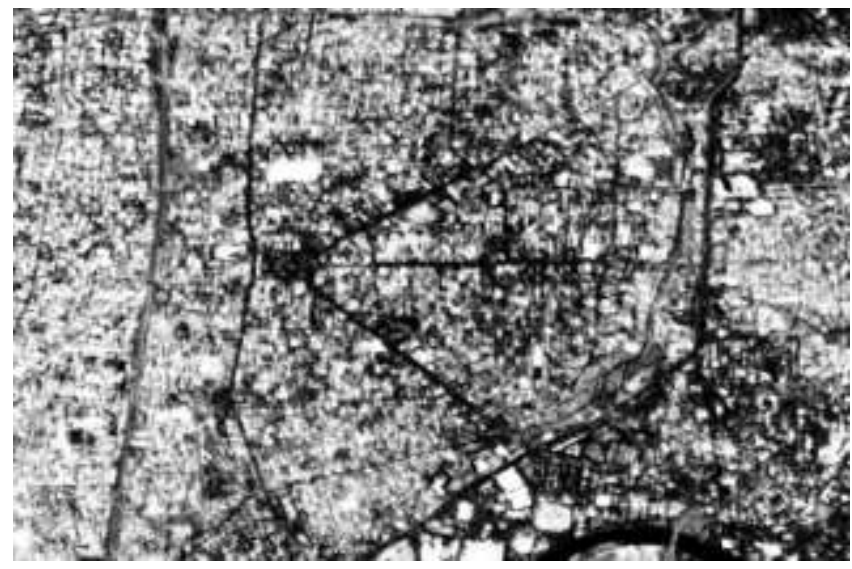

"Figure 11" Scanned Satellite Image

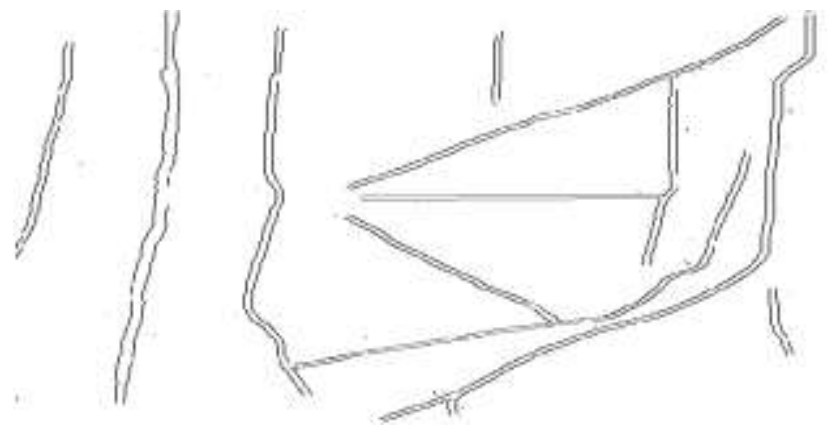

“Figure12" Skeletonization by Mathematical Morphology

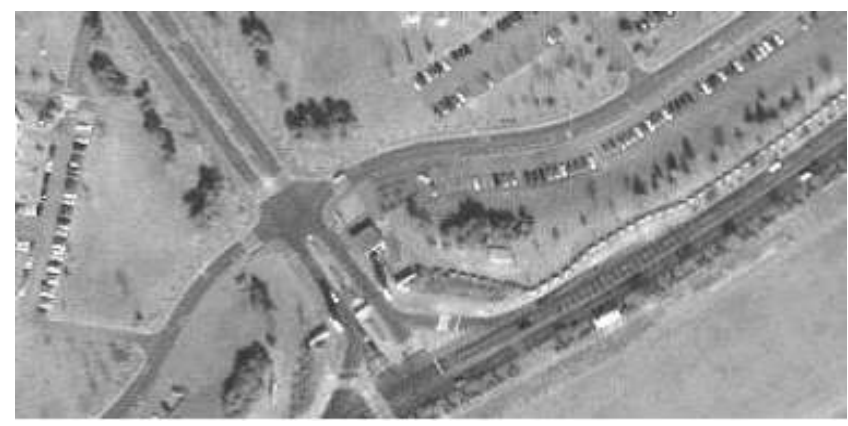

“Figure13" Scanned Satellite image

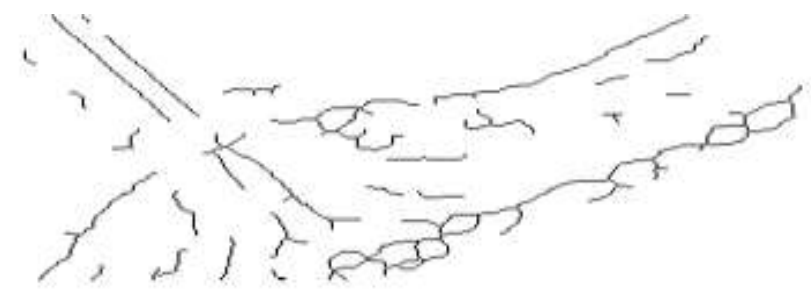

“Figure14" Skeletonization by Mathematical Morphology

\section{Conclusion}

The obtained results assisted the objectives about the use of the tools of the Mathematical Morphology in the improvement process of the visual quality of the original images and the extraction of the obtained products through remote sensing. It is worth to stand out that the choice of the operators and appropriate thresholds contributed to the extraction of the features, in which the results indicate that the employed morphologic processing is appropriate.

Although the adopted thresholds based on the analysis of the Outsu algorithm provides best results (see figure 11 and figure 12) are split and the different contour levels are written in the gap. This causes problems in automatic vectorization of images.

The gap filling methods will be describes in the next paper.

\section{References}

[1] Anonymous 2008. Wikipedia, the free encyclopedia on Mathematical Morphology [Online].Available at http://en.wikipedia.org/w/index.php?title=Mathematical morphology.

[2] Daya Bhavya 2008. Parallelization of Two-Dimensional Skeletonization Algorithms. Journal of Undergraduate Research,University of florida. Volume 9, Issue 4,pp.

[3] Alan Peters II Richard 2007. Lecture Notes on Mathematical Morphology EECE/CS 253 image Processing. Department of Electrical Engineering and Computer science, Vanderbilt University School of Engineering San Francisco, California,pp-11,18,25.(Fig of D,E).

[4] Rafael C. Gonzalez, Richard E. Woods, Steven L. Eddins 2007. Digital Image Processing using MATLAB. Pearson Prentice hall, Pearson Education Inc.

[5] Salvatore S., Guitton P. 2004. Contour line recognition from scanned topographic images. Journal of WSCG.

[6] Gottfried Konecny. Geoinformation 2003. Remote Sensing, Photogrammetry and Geographic Information Systems. Taylor and Francis Inc.

[7] Wu S., Amin A. 2003.Automatic thresholding of gray-level using multi-stage approach. In IEEE, Proceedings of the Seventh International Conference on Document Analysis and Recognition, pp 493-497.

[8] Weiss John 2002.Grayscale thinning. In Proceedings of the 17th International Conference on Computers and Their Applications (CATA-2002), pp 86-89.

[9] Tombre K., Tabbone S. 2000. Vectorization in graphics recognition: to thin or not to thin. Volume 2 of Proceedings of 15th International Conference on Pattern Recognition of IEEE, pp 91-96. 
[10] Wiedmann C.,Hinz S. 1999. Automatic extraction and evaluation of road networks from satellite imagery. In $\mathrm{H}$. Ebner, W. Eckstein, C. Heipke, and H. Mayer, editors, Proceedings ISPRS Conference on Automatic Extraction of GIS Objects from Digital Imagery, Vol. XXXII/3-2W5 of International Archives of Photo grammetry and Remote Sensing, pp 95-100.

[11] Liu W, Dori D. 1997. A Protocol for Performance Evaluation of Line Detection Algorithms. Machine Vision Applications; 9:240-250.

[12] Susan Mayhew 1997. Dictionary of Geography.Oxford University Press.
[13] J. R. Munkres 1993.Elements of Algebraic Topology. Perseus Press.

[14] Nalwa VS 1993.A Guided Tour of Computer Vision. Addison-Wesley, New York.

[15] Boatto L et al 1992. An Interpretation System for Land Register Images. IEEE Computer; 25(7):25-32.

[16] Watson L.T., Arvind K., Ehrich R.W., Haralick R.M. 1984. Extraction of lines and regions from greytone line drawing images. Pattern Recognition; 17:493-507. 